\title{
La gamificación en el ámbito educativo español: revisión sistemática Gamification in the Spanish educational field: a systematic review
}

\author{
Carmen Navarro-Mateos, Isaac José Pérez-López, Pedro Femia Marzo
}

Universidad de Granada (España)

\begin{abstract}
Resumen. Ante la falta de motivación e implicación del alumnado surgen nuevas estrategias metodológicas que acercan los procesos educativos a los intereses y a las necesidades del estudiante del siglo XXI. Entre ellas destaca la gamificación, que consiste en aplicar los elementos propios del juego en contextos no lúdicos. Esta revisión sistemática pretende conocer la incidencia que la gamificación ha alcanzado en el ámbito educativo español para poder determinar en qué etapas y en qué asignaturas se está aplicando, sobre qué variables se incide y qué resultados se están obteniendo. Para ello se consultaron las bases de datos electrónicas Web of Science (colección principal), Eric y Scopus, contemplando los principios establecidos por la declaración PRISMA para revisiones sistemáticas. De los 118 artículos cribados por el título y el resumen y leídos en su totalidad, solo 15 propuestas cumplieron los criterios de inclusión. En la distribución por etapas educativas el 66.7\% de los artículos pertenecen al ámbito universitario, el $20 \%$ a educación secundaria, el 6,7\% a bachillerato y el 6,7\% restante combinaba el programa en primaria y secundaria. En términos globales, más de la mitad de las propuestas (un 60\%) incidieron en variables motivacionales. A pesar del enorme auge de la gamificación en los últimos años, se ha identificado la necesidad de un mayor rigor metodológico y una descripción más exhaustiva de las propuestas para valorar su verdadera potencialidad en nuestro país.
\end{abstract}

Palabras clave: gamificación, educación, aprendizaje, motivación, instituto, bachillerato, universidad.

Abstract. In order to face the lack of motivation and involvement of the student body new methodological strategies have emerged aiming to bring the educational processes closer to the interests and necessities of the 21st century's students. Among them, gamification stands out as a strategy that consists of applying elements from the games in non-ludic contexts. This systematic review seeks to know the impact that gamification has reached in the Spanish educational field to determine in which stages and which courses it is being applied to, on which variables it has an impact on and what are the results that we are obtaining. In order to do this, we consulted digital databases such as: Web of Science (main collection), Eric and Scopus. The principles established by the PRISMA declaration were taken into consideration. Out of the 118 articles filtered by title and abstract, and then read in its entirety only 15 proposals fulfilled the inclusion criteria. In the distribution via different education stages, $66.7 \%$ of the articles fall into the category of university education, $20 \%$ belongs to mandatory high school education, $6.7 \%$ falls into the category of Spanish baccalaureate and the remaining 6.7\% combined the program on mandatory primary school and secondary school education. If we think in global terms, more than half of the proposals (60\%) had an impact on motivational variables. Despite the rise of gamification in the last few years it has been identified a necessity for a higher methodological exactitude and a more exhaustive description of proposals to value their true potentiality in our country.

Key words: gamification, education, learning process, motivation, high school, Spanish baccalaureate, university.

\section{Introducción}

En una sociedad en continua transformación, la educación es una de las áreas que se enfrenta a más cambios y retos para dar respuesta a las necesidades del alumnado, considerándose el conjunto de elementos que constituyen el sistema educativo actual como obsoletos al no llegar a adaptarse a las demandas actuales (GiménezGiubbani, 2016; Severín, 2017). Uno de los aspectos que mayor incidencia tiene en dicha circunstancia es

Fecha recepción: 28-01-21. Fecha de aceptación: 28-04-21

Carmen Navarro Mateos

carmenavarro@correo.ugr.es que las instituciones educativas se preocupan habitualmente más por los resultados que por los procedimientos, más por lo que los docentes enseñan que por lo que los estudiantes aprenden, más por la calificación que por la evaluación (Cornellà \& Estebanell, 2018). Las respuestas a los problemas actuales tienden a sostener una mirada retrospectiva más que a inventar una propuesta prospectiva (Ortega-Navas, 2016). Esta realidad provoca falta de compromiso e implicación en el alumnado al no considerar lo que aprende como significativo, provocándole una enorme desmotivación (Bolívar, 2018; Torres, 2016; Zepeda-Hernández, Abascal-Mena \& López-Ornelas, 2016). En la línea de autores como Chrobak (2017), Giménez-Giubbani (2016) o Jiménez- 
Serrano (2002), se debe propugnar una enseñanza centrada sobre la actividad autónoma del alumno, con propuestas curriculares más adaptadas a los requerimientos de la vida en sociedad, conectando con el entorno en el que el alumno se desarrolla y dando respuestas a las necesidades del futuro. De esta manera se acercará la vida a la escuela (Perrenoud, 2012), provocando la aplicación de conocimientos en situaciones reales, construyendo una educación auténtica, es decir «situada», que contextualice los conocimientos sin perder de vista el todo (Tobón, Guzmán, Silvano-Hernández \& Cardona, 2015). Lo anteriormente comentado trae consigo la responsabilidad de profesores e instituciones a la hora de innovar en metodologías emergentes que intenten incorporar en sus clases estrategias que hagan protagonista al alumnado (Garrote, Garrote \& Jiménez-Fernández, 2016; López-López, 2019), que aumenten su motivación y compromiso, y que les proporcionen las herramientas y recursos necesarios para favorecer el aprendizaje autónomo y significativo (Chrobak, 2017; De Soto-García, 2018; Ortiz-Colón, Jordán \& Agredal, 2018).

En este sentido, una poderosa estrategia para motivar y favorecer el aprendizaje del alumnado es la gamificación, entendida como el uso de elementos lúdicos en contextos que no son de juego (Deterding, 2011). Esta se caracteriza por incrementar el interés e implicación del alumnado en los procesos de enseñanza-aprendizaje (Hanus \& Fox, 2015; Kapp, 2012; Llopis \& Balaguer, 2017; Zichermann \& Cunningham, 2011). Se han realizado propuestas diversas para gamificar sectores muy diferentes: empresas, recursos humanos, entrenamientos, salud, comunicación, marketing, desarrollo de productos... (Bonilla, 2015; Chang et al., 2018; Hamari, 2013; Hamari, Koivisto \& Sarsa, 2014). En el ámbito educativo también se están llevando a cabo, cada vez con mayor frecuencia, pudiendo encontrar diversos autores internacionales que inciden en su potencial (Arnold, 2014; Buckley, Doyle \& Doyle, 2017; Lee \& Hammer, 2011).

Sin embargo, al ser un concepto emergente, la literatura relacionada con esta temática es relativamente reciente, interdisciplinaria y de naturaleza heterogénea (Lumsden, Edwards, Lawrence, Coyle \& Munafó, 2016). En el caso de España, son escasos los trabajos existentes en la literatura científica que analicen el impacto de la gamificación en el sistema educativo. Además, la mayoría de ellos, se centran únicamente en una determinada etapa educativa como, por ejemplo, educación secundaria (Parra-González \& Segura-Robles, 2019) o en una asignatura concreta, como puede ser educación física (León-Díaz, Martínez-Muñoz \& Santos-Pastor, 2019).

Para que el verdadero potencial de la gamificación pueda hacerse realidad en este ámbito, como señala Kapp (2012), no puede reducirse básicamente a establecer una estructura de recompensas según determinadas acciones realizadas por los jugadores (alumnos). En el ámbito educativo, desafortunadamente, en numerosas ocasiones se atribuye el concepto de gamificación a experiencias que solamente utilizan puntos en lugar de notas y que se combinan con insignias y clasificaciones (Kapp, 2012). Esto es lo que se conoce como PBL (points, badges and leaderboards) y corresponde a experiencias de gamificación de «capa fina», aquellas que tendrán poca repercusión en el aprendizaje al apostar más por la motivación extrínseca que por la intrínseca y que, además, se llevan a cabo en periodos cortos de tiempo (Marczewski, 2018). Estos elementos deben ir acompañados de aquellos otros que requieren la implicación de los participantes como, por ejemplo, la narrativa, las misiones, los niveles etc., y que son los cimientos sobre los que la gamificación debe construirse para que la motivación extrínseca pueda llegar a convertirse en intrínseca (Dichev, Dicheva, Angelova \& Agre, 2014; Faiella \& Ricciardi, 2015; Toledo, Toda, Oliveira, Cristea \& Isotani, 2019). La conexión emocional a través de una narrativa posee un fuerte componente motivacional e inmersivo, captando la atención del alumnado para desarrollar un aprendizaje en un ambiente agradable y creativo (Quintero-González, Jiménez-Jiménez \& AreaMoreira, 2018). Todo ello permite convertir el proceso de enseñanza-aprendizaje en un elemento nuevo, atractivo y, en última instancia, mucho más efectivo (Torres, Romero-Rodríguez, Pérez-Rodríguez \& Björk, 2018).

En estrecha relación con ello, es preciso destacar que el concepto de gamificación suele confundirse con otros términos que tienen también el juego como referencia, en parte debido a las pobres definiciones de los términos existentes (Lumsden et al., 2016). El más habitual es el de aprendizaje basado en juegos (ABJ), observándose en la literatura un uso indistinto entre los términos de gamificación y ABJ (Monguillot, GonzálezArévalo, Zurita, Almirall \& Guitert, 2015; ParraGonzález \& Segura-Robles, 2019). El ABJ consiste en la utilización de juegos y videojuegos como vehículos y herramientas para impulsar al aprendizaje, la asimilación o la evaluación de conocimientos (Del Moral, Guzmán-Duque \& Fernández-García, 2018), mientras que la gamificación usa las mecánicas y dinámicas de los juegos, pero no se articula en un juego en sí (Gómez- 
Gonzalvo, Molina-Alventosa \& Devis, 2018; Marín, 2018).

Es por ello que el objetivo de esta revisión sistemática es conocer la incidencia que la gamificación ha alcanzado en el ámbito educativo español a través del análisis de todas aquellas intervenciones realizadas hasta el momento actual, sin restricción de asignaturas ni etapas educativas. De esta manera, se podrá valorar si la gamificación ha alcanzado en España la misma repercusión que fuera de sus fronteras (Majuri, Koivisto \& Hamari, 2018; Nah, Zeng, Telaprolu, Ayyappa \& Eschenbrenner, 2014). A raíz de ello se determinará en qué etapas y en qué asignaturas se está aplicando, sobre qué variables se está incidiendo, qué herramientas se utilizan para medir dichas variables y qué resultados se están obteniendo. Además, el resultado de esta revisión sistemática aportará un material de gran valía a los docentes que decidan incluir la gamificación en sus programaciones.

\section{Método}

Para la realización de esta revisión sistemática se consultaron las siguientes bases de datos electrónicas: Web of Science (colección principal), Eric y Scopus. La estrategia de búsqueda (tabla 1) combina texto libre y tesauros incluidos en Eric. Con estas palabras claves se contemplaban las propuestas gamificadas realizadas en educación infantil o preescolar, en el colegio (al utilizar el término «school» también se incluían aquellas realizadas en preescolar, «preschool»), en instituto, en universidad (se utilizó «*» para contemplar los sufijos posibles) y en formación profesional. Incluía artículos y congresos publicados en España y cuyo idioma fuese el español o el inglés. En los casos en los que hubiese un artículo y congreso que tratara el mismo proyecto, se seleccionaba el artículo. Esta búsqueda se cerró el 31 de diciembre de 2020, incluyendo todas las intervenciones realizadas hasta la fecha.

Tabla 1

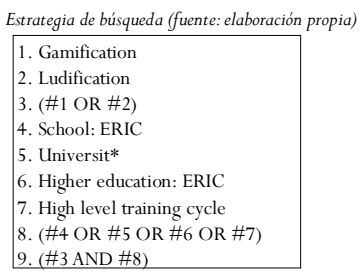

\section{Criterios de inclusión y exclusión}

Se han incluido todas las propuestas gamificadas:

- Realizadas en España.

- Llevadas a cabo en cualquier nivel educativo de manera formal y presencial.

- Encuadradas en alguna asignatura del currículo correspondiente a la etapa educativa en cuestión.

- Con incidencia en algún aprendizaje concreto del currículo o en alguna variable que incidiese en dicho aprendizaje.

- Que hayan utilizado instrumentos de evaluación para medir el impacto de la misma.

Se excluyeron aquellas intervenciones que se limitaban al PBL o que usaban exclusivamente aplicaciones o plataformas. La revisión también excluyó resúmenes, revisiones narrativas, revisiones sistemáticas y metaanálisis.

\section{Resultados de la búsqueda}

Se realizó la búsqueda en las bases de datos anteriormente citadas, analizándose en un primer momento el título, el resumen y las palabras claves. Las propuestas relevantes y potencialmente válidas fueron seleccionadas y clasificadas para su lectura íntegra. Aquellas cuya información no era suficiente para tomar una decisión también fueron seleccionadas y clasificadas para su posterior análisis. Las propuestas que no cumplían los criterios citados anteriormente fueron descartadas.

En base a los principios establecidos por la declaración PRISMA (Preferred Reporting Items for Systematic reviews and Meta-Analyses -figura 1-) para la elaboración de revisiones sistemáticas, se identificaron un total de 408 registros en las tres bases de datos que, tras descartar los registros duplicados (98) se quedaron en 310. De ellos, 192 fueron eliminados tras la lectura del título y del resumen. De los 118 artículos resultantes se realizó la lectura íntegra del artículo para comprobar si verdaderamente se cumplían o no los criterios de inclusión. El proceso terminó con un total de 15 artículos incluidos en esta revisión sistemática.

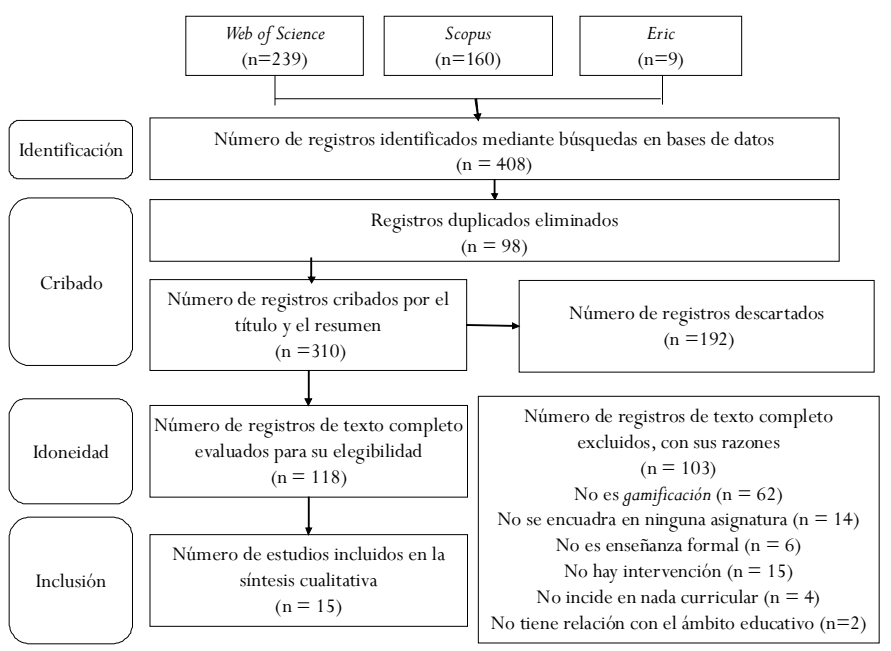

Figura 1. Diagrama de flujo de la selección de estudios (fuente elaboración propia) 
De los 62 registros que se han excluido por no ser gamificación (figura 1), 24 se centraban en el uso de aplicaciones y plataformas (como Kahoot!, Socrative o Classcraft), 18 de ellos eran ABJ, 15 PBL y, por último, cuatro eran ABP (aprendizaje basado en problemas) y uno ApS (aprendizaje servicio).

\section{Resultados}

Con los datos obtenidos (tabla 2), la distribución por etapas educativas de las propuestas identificadas resultante es del $66.7 \%$ en el ámbito universitario, el 20\% en la ESO (enseñanza secundaria obligatoria), el 6.7\% en bachillerato y en el $6.7 \%$ restante se lleva a cabo la intervención tanto en primaria como en secundaria. Dentro del ámbito universitario, tres de ellas se ubican en grados de ingeniería, cinco en el de ciencias del deporte y dos en el de educación primaria.

En las ingenierías algunas de las variables sobre las que se han incidido han sido el compromiso, la motivación o la mejora de la experiencia de aprendizaje (SousaVieira, López-Ardao, Fernández-Veiga, RodríguezPérez \& López-García, 2015; Vicent, Villagrasa, Fonseca \& Redondo, 2015; Villagrasa, Fonseca, Redondo \& Durán, 2014), como consecuencia de la alta desmotivación y los niveles de absentismo.

En el grado de ciencias del deporte, sin embargo, aprovechan la gamificación para mejorar los hábitos de vida saludables, el clima de aula, la asimilación de los aprendizajes, el fitness cardiorrespiratorio o la gestión emocional (Mora-González, Pérez-López \& DelgadoFernández, 2020; Mora-González, Pérez-López, Esteban-Cornejo \& Delgado-Fernández, 2020; Pérez-López, Rivera \& Delgado, 2017; Pérez-López, Rivera \& Trigueros, 2017; Pérez-López, Rivera \& Trigueros, 2019).

Con relación al grado de educación primaria, los principales objetivos están relacionados con variables como el rendimiento académico, el trabajo colaborativo o la motivación (Cornellà \& Estebanell, 2018; SánchezMartín, Cañada-Cañada \& Dávila-Acedo, 2017).

Respecto a las propuestas realizadas en el resto de etapas educativas, cuatro de las cinco existentes tuvieron lugar en la asignatura de educación física (dos en ESO, una que combinaba alumnos de primaria y secundaria y otra en bachillerato), y la quinta (también en ESO) tuvo lugar en la asignatura de lengua castellana. Todas ellas tenían como objetivo incidir en la motivación del alumnado (Fernández-Río, De las Heras, González, Trillo \& Palomares, 2020; Martín-Moya, Ruiz-
Montero, Chiva-Bartoll \& Capella-Peris, 2018; Menchaca, Moya \& Bastida, 2019; Monguillot et al., 2015; Valero-Valenzuela, García, Camerino \& Manzano, 2020) y, además, en una de las intervenciones se trataron parámetros relacionados con la salud, como es la frecuencia cardíaca saludable (Monguillot et al., 2015).

En términos globales, más de la mitad de las propuestas (un 60\%) incidieron en variables motivacionales. Cinco de ellas han utilizado herramientas concretas para cuantificar el cambio de conducta, concluyendo todas con resultados positivos (Fernández-Río et al., 2020; Martín-Moya et al., 2018; Valero-Valenzuela et al., 2020; Vicent et al., 2015; Villagrasa et al., 2014). Además, en tres de los trabajos, tras el correspondiente tratamiento de los datos, existía correlación positiva entre las notas académicas y los logros conseguidos en el entorno gamificado, incrementando el rendimiento académico (Sánchez-Martín et al., 2017; Sousa-Vieira et al., 2015; Vicent et al., 2015).

En lo que respecta a los instrumentos de medida, en cuatro propuestas se han combinado instrumentos cuantitativos y cualitativos para conocer el grado de satisfacción o valoración del alumnado sobre la propuesta, constrastándolos con los aprendizajes adquiridos. Mientras tanto, en el 25\% se han utilizado únicamente herramientas cualitativas a diferencia del $44 \%$ de las intervenciones que han sido evaluadas de forma cuantitativa.

Si se pone el foco en la duración de los programas se aprecia una gran disparidad, oscilando entre una duración de 10 sesiones y un semestre. Por último, llama la atención que atendiendo a la distribución geográfica, más de dos tercios de las intervenciones se ubican en dos ciudades concretas, Barcelona (Cataluña) y Granada (Andalucía).

\section{Discusión}

Tras la revisión realizada solo se han identificado 15 propuestas de gamificación en el ámbito educativo español. Este reducido número llama la atención, teniendo en cuenta lo que sucede en el ámbito internacional, donde su repercusión es mucho mayor (Dicheva, Dichev, Agre \& Angelova, 2015; Mora, Riera, González \& Arnedo-Moreno, 2017; Nah et al., 2014; Zainuddin, Chu, Shujahat \& Perera, 2020). Además, únicamente, se encuentra una propuesta que cumpla con los criterios de inclusión que haga parte de su intervención en educación primaria. Dicha circunstancia llama la atención, ya que el enfoque lúdico en general está integrado de for- 
Intervenciones gamificadas llevadas a cabo en el ámbito educativo español (fuente: elaboración propia)

\begin{tabular}{|c|c|c|c|c|c|}
\hline Referencia & $\begin{array}{l}\text { Nivel educativo } \\
\text { (comunidad } \\
\text { autonónoma) }\end{array}$ & $\begin{array}{l}\text { Curso, } \\
\text { asignatura, duración y tamaño de muestra }\end{array}$ & Variable en la que incide & Instrumento de medida & Tratamiento de los datos y resultados \\
\hline $\begin{array}{l}\text { Villagrasa, Fonseca, } \\
\text { Redondo \& Durán } \\
\text { (2014) }\end{array}$ & $\begin{array}{l}\text { Universidad } \\
\text { Barcelona } \\
\text { (Cataluña) }\end{array}$ & $\begin{array}{c}2^{\circ} \text { curso del grado de Ingeniería Multimedia } \\
\text { Animación por ordenador I } \\
30 \text { semanas } \\
\mathrm{n}=\text { ? }\end{array}$ & $\begin{array}{l}\text { Motivación } \\
\text { Compromiso }\end{array}$ & $\begin{array}{c}\text { Cuestionario de eficacia y } \\
\text { eficiencia } \\
+ \\
\text { Sistema BLA } \\
\text { (bipolar laddering) }\end{array}$ & $\begin{array}{l}\text { Polarización según los criterios (positivo/negativo- } \\
\text { elementos comunes) } \\
\text { Un mejor rendimiento académico que se atribuye a las } \\
\text { técnicas de gamificación y al resto de metodologías } \\
\text { utilizadas. } \\
\text { Los alumnos aumentaron su motivación }\end{array}$ \\
\hline $\begin{array}{l}\text { Monguillot, González- } \\
\text { Arévalo, Zurita, } \\
\text { Almirall \& Guitert } \\
\text { (2015) }\end{array}$ & $\begin{array}{l}\text { Secundaria } \\
\text { Barcelona } \\
\text { (Cataluña) }\end{array}$ & $\begin{array}{l}2^{\circ} \mathrm{ESO} \\
\text { Educación Física } \\
12 \text { sesiones } \\
\mathrm{n}=99\end{array}$ & $\begin{array}{c}\text { Motivación } \\
\text { Aplicación de la } \\
\text { frecuencia cardíaca } \\
\text { saludable (FCS) en tareas } \\
\text { de resistencia aeróbica }\end{array}$ & $\begin{array}{c}\text { Observación participante } \\
+ \\
\text { Cuestionario } \\
+ \\
\text { Grupo de discusión o entrevista } \\
\text { grupal }\end{array}$ & $\begin{array}{c}\text { El } 77 \% \text { de alumnado ha valorado la gamificación como una } \\
\text { estrategia de motivación para el aprendizaje de conductas } \\
\text { saludables y el } 98 \% \text { han afirmado haber aprendido a } \\
\text { aplicar la FCS }\end{array}$ \\
\hline $\begin{array}{l}\text { Sousa-Vieira, López- } \\
\text { Ardao, Fernández- } \\
\text { Veiga, Rodríguez-Pérez } \\
\text { \& López-García (2015) }\end{array}$ & $\begin{array}{l}\text { Universidad } \\
\text { Vigo } \\
\text { (Galicia) }\end{array}$ & $\begin{array}{c}\text { Ingeniería } \\
\text { Redes de Computadores } \\
14 \text { semanas } \\
\mathrm{n}=86\end{array}$ & $\begin{array}{l}\text { Motivación } \\
\text { Mejora de la experiencia } \\
\text { de aprendizaje y } \\
\text { rendimiento }\end{array}$ & $\begin{array}{c}\text { Calificaciones } \\
+ \\
\text { Encuesta de satisfacción con la } \\
\text { plataforma y la metodología }\end{array}$ & $\begin{array}{c}\text { Prueba T de Student } \\
\text { Test de regresión lineal } \\
\text { Test de Kendall } \\
\text { Dependencia positiva significativa }(p<.05) \text { entre las } \\
\text { calificaciones de las actividades de evaluación continua } \\
\text { (examen de mitad de período y las tareas de } \\
\text { programación), los puntos de juego en las actividades } \\
\text { sociales y la nota final }\end{array}$ \\
\hline $\begin{array}{l}\text { Vicent, Villagrasa, } \\
\text { Fonseca \& Redondo } \\
\text { (2015) }\end{array}$ & $\begin{array}{l}\text { Universidad } \\
\text { Barcelona } \\
\text { (Cataluña) }\end{array}$ & $\begin{array}{c}2^{\circ} \text { curso del grado en Ingeniería de Edificación } \\
\text { Herramientas de Dibujo II } \\
16 \text { semanas } \\
\mathrm{n}=65\end{array}$ & $\begin{array}{c}\text { Motivación } \\
\text { Rendimiento académico }\end{array}$ & $\begin{array}{c}\text { Sistema BLA (bipolar laddering) } \\
+ \\
\text { Comparación de notas }\end{array}$ & $\begin{array}{l}\text { Polarización según los criterios (positivo/negativo- } \\
\text { elementos comunes) } \\
\text { Incremento del rendimiento académico y de la } \\
\text { motivación }\end{array}$ \\
\hline $\begin{array}{l}\text { Pérez-López, } \\
\text { Rivera \& Delgado- } \\
\text { Fernández (2017) }\end{array}$ & $\begin{array}{l}\text { Universidad } \\
\text { Granada } \\
\text { (Andalucía) }\end{array}$ & $\begin{array}{c}3^{\circ} \text { curso del grado de Ciencias del Deporte } \\
\text { Actividad Física y Salud } \\
\text { Un cuatrimestre } \\
\mathrm{n}=148\end{array}$ & $\begin{array}{l}\text { Hábitos de vida } \\
\text { saludables }\end{array}$ & $\begin{array}{c}\text { "Encuesta Verde" } \\
+ \\
\text { Análisis cualitativo de la } \\
\text { valoración a través de un } \\
\text { cuestionario de Google }\end{array}$ & $\begin{array}{l}\text { ANCOVA y análisis cualitativo (software NVivolo) } \\
\text { Mejora muy significativa del valor global medio de los } \\
\text { hábitos de vida saludables del grupo experimental } \\
\text { respecto al grupo control }(p<.001)\end{array}$ \\
\hline $\begin{array}{l}\text { Pérez-López, Rivera \& } \\
\text { Trigueros (2017) }\end{array}$ & $\begin{array}{l}\text { Universidad } \\
\text { Granada } \\
\text { (Andalucía) }\end{array}$ & $\begin{array}{l}4^{\circ} \text { curso del grado de Ciencias del Deporte } \\
\text { Un semestre } \\
\text { Enseñanza de la Actividad Física y el Deporte } \\
n=69\end{array}$ & $\begin{array}{c}\text { Clima de aula } \\
\text { Aprendizajes adquiridos }\end{array}$ & $\begin{array}{l}\text { Narraciones evaluativas de los } \\
\text { estudiantes a través de un } \\
\text { formulario de Google }\end{array}$ & $\begin{array}{l}\text { Análisis cualitativo (software } \mathrm{NVivo} 10 \text { ) } \\
\text { Clima de aula distendido, sensación de control y asunción } \\
\text { de responsabilidad y adquisición de recursos de segundo } \\
\text { nivel (deontología profesional, compromiso valórico y } \\
\text { social y construcción de su identidad) }\end{array}$ \\
\hline $\begin{array}{l}\text { Sánchez-Martín, } \\
\text { Cañada \& Dávila } \\
\text { (2017) }\end{array}$ & $\begin{array}{l}\text { Universidad } \\
\text { Badajoz } \\
\text { (Extremadura) }\end{array}$ & $\begin{array}{c}2^{\circ} \text { curso del grado de Educación Primaria } \\
\text { Materia y Energía } \\
\text { Dos meses } \\
\mathrm{n}=36\end{array}$ & $\begin{array}{l}\text { Rendimiento académico } \\
\text { Trabajo colaborativo }\end{array}$ & $\begin{array}{l}\text { Recuento de puntos e índice de } \\
\text { juego }\end{array}$ & $\begin{array}{l}\text { Análisis descriptivo de los resultados finales del juego y } \\
\text { ANOVA } \\
\text { Correlación positiva entre las notas académicas y las } \\
\text { puntuaciones en el juego }\left(\mathrm{R}^{2}=72 \%, p^{<}<.001\right) \\
\text { Invirtieron sus puntos en ganancias individuales, no se } \\
\text { implementaron medidas colectivas }\end{array}$ \\
\hline $\begin{array}{l}\text { Cornellà \& Estebanell } \\
\quad \text { (2018) }\end{array}$ & $\begin{array}{l}\text { Universidad } \\
\text { Girona } \\
\text { (Cataluña) }\end{array}$ & $\begin{array}{c}4^{\circ} \text { curso del grado de Educación Primaria } \\
\text { Videojuegos y Educación } \\
\text { Un semestre } \\
\mathrm{n}=97\end{array}$ & Motivación & $\begin{array}{l}\text { Cuestionario para valorar } \\
\text { diversos aspectos de la } \\
\text { gamificación en un entorno } \\
\text { Moodle } \\
\end{array}$ & $\begin{array}{c}\text { Los estudiantes valoran positivamente tanto la aplicación } \\
\text { de la gamificación en su proceso de aprendizaje, como el } \\
\text { uso de Moodle como herramienta para dar soporte a esta } \\
\text { metodología }\end{array}$ \\
\hline $\begin{array}{l}\text { Martín-Moya, Ruiz- } \\
\text { Montero, Chiva-Bartoll } \\
\text { \& Capella-Peris } \\
\text { (2018) }\end{array}$ & $\begin{array}{l}\text { Bachillerato } \\
\text { Granada } \\
\text { (Andalucía) }\end{array}$ & $\begin{array}{l}2^{\circ} \text { de Bachillerato } \\
\text { Educación Física } \\
13 \text { sesiones } \\
\mathrm{n}=30\end{array}$ & $\begin{array}{c}\text { Variaciones } \\
\text { motivacionales }\end{array}$ & $\begin{array}{c}\text { Cuestionario socio-demográfico } \\
+ \\
\text { Versión española del } \\
\text { "Achievement Motivation for } \\
\text { Learning in Physical Education" } \\
\text { (AMPET) }\end{array}$ & $\begin{array}{c}\text { Test de Wilcoxon } \\
\text { o } \\
\text { Resultados significativos en todas las dimensiones } \\
\text { respecto al total grupo-clase; la dimensión del } \\
\text { compromiso con el aprendizaje en el grupo de alumnos } \\
(p=.004), \text { y la percepción de competencia motriz } \\
\text { autopercibida }(p=.042) \text { y la ansiedad y agobio ante el } \\
\text { fracaso en el total grupo-clase }(p=.007) \text { en el grupo de } \\
\text { alumnas }(p=.131)\end{array}$ \\
\hline $\begin{array}{l}\text { Menchaca, Moya \& } \\
\text { Bastida (2019) }\end{array}$ & Secundaria & $\begin{array}{l}2^{\circ} \text { ESO } \\
\begin{array}{c}\text { Lengua Castellana y Literatura } \\
22 \text { sesiones } \\
\mathrm{n}=20\end{array}\end{array}$ & $\begin{array}{c}\text { Motivación } \\
\text { Trabajo en equipo }\end{array}$ & $\begin{array}{c}\text { Control de asistencia } \\
+ \\
\text { Cuestionarios pre y post } \\
+ \\
\text { Análisis de las notas finales }\end{array}$ & $\begin{array}{c}\text { Análisis cualitativo } \\
\text { (software NVivo 11 Plus) } \\
\text { 100\% de asistencia, mejora de la motivación, aumento de } \\
\text { la media de la calificación del examen ( } 0 \% \text { de suspensos) } \\
\text { y mejora de la motivación de la profesora }\end{array}$ \\
\hline $\begin{array}{l}\text { Pérez-López, Rivera \& } \\
\text { Trigueros (2019) }\end{array}$ & $\begin{array}{l}\text { Universidad } \\
\text { Granada } \\
\text { (Andalucía) }\end{array}$ & $\begin{array}{c}2^{\circ} \text { curso del grado de Ciencias del Deporte } \\
\text { Fundamentos de la Educación Física } \\
\text { Un cuatrimestre } \\
\mathrm{n}=59\end{array}$ & $\begin{array}{l}\text { Sentimientos/gestión } \\
\text { emocional }\end{array}$ & $\begin{array}{c}\text { Cuestionario abierto de Google } \\
\text { Drive }\end{array}$ & $\begin{array}{c}\text { Análisis cualitativo } \\
\text { (software NVivo 11 Plus) } \\
\text { Mejora de la motivación e implicación. Sentimiento de } \\
\text { satisfacción a lo largo de todo el proceso }\end{array}$ \\
\hline $\begin{array}{l}\text { Fernández-Río, De las } \\
\text { Heras, González, Trillo } \\
\text { \& Palomares (2020) }\end{array}$ & $\begin{array}{l}\text { Primaria y } \\
\text { secundaria } \\
\text { Cuatro centros en } \\
\text { cuatro regiones } \\
\text { diferentes (urbano- } \\
\text { rural y nivel } \\
\text { socioeconómico } \\
\text { bajo-medio-alto) }\end{array}$ & $\begin{array}{l}\text { De } 2^{\circ} \text { primaria a } 2^{\circ} \text { ESO } \\
\text { Educación Física } \\
15 \text { semanas ( } 30 \text { sesiones) } \\
n=290\end{array}$ & Motivación intrínseca & $\begin{array}{c}\text { Subescala de motivación } \\
\text { intrínseca (versión española) } \\
+ \\
\text { Análisis cualitativo (dibujos, } \\
\text { grupos de discusión y diario del } \\
\text { profesor) }\end{array}$ & $\begin{array}{l}\text { ANOVA y análisis cualitativo (MAXQDA 11) } \\
\text { Mejora significativa de la motivación intrínseca }(p>01) \\
\text { basándonos en el nivel. Con respecto a los dibujos el } \\
\text { tema principal fue el disfrute y los secundarios los amigos } \\
\text { y el aprendizaje. Las respuestas más repetidas entre los } \\
\text { profesores: carga de trabajo, portfolio y narrativa }\end{array}$ \\
\hline $\begin{array}{l}\text { Mora-González, Pérez- } \\
\text { López \& Delgado- } \\
\text { Fernández (2020) }\end{array}$ & $\begin{array}{l}\text { Universidad } \\
\text { Granada } \\
\text { (Andalucía) }\end{array}$ & $\begin{array}{c}2^{\circ} \text { curso del grado de Ciencias del Deporte } \\
\text { Fundamentos de la Educación Física } \\
\text { Un cuatrimestre } \\
\mathrm{n}=117\end{array}$ & $\begin{array}{c}\text { Fitness } \\
\text { cardiorrespiratorio }\end{array}$ & $\begin{array}{c}\text { Test de } 20 \text { metros ida y vuelta } \\
+ \\
\text { Runtastic }\end{array}$ & $\begin{array}{c}\text { ANCOVA } \\
\text { Mejora significativa para el grupo intervención en el } \\
\text { número de estadios completados en el test de } 20 \text { metros } \\
(p<.001) \text { y en la estimación del VO2máx }(p<.001) \\
\end{array}$ \\
\hline $\begin{array}{l}\text { Mora-González, Pérez- } \\
\text { López, Esteban- } \\
\text { Cornejo \& Delgado- } \\
\text { Fernández (2020) }\end{array}$ & $\begin{array}{l}\text { Universidad } \\
\text { Granada } \\
\text { (Andalucía) }\end{array}$ & $\begin{array}{c}2^{\circ} \text { curso del grado de Ciencias del Deporte } \\
\text { Fundamentos de la Educación Física } \\
\text { Un cuatrimestre } \\
\mathrm{n}=112\end{array}$ & $\begin{array}{c}\text { Fitness } \\
\text { cardiorrespiratorio }\end{array}$ & Test de 20 metros ida y vuelta & $\begin{array}{c}\text { ANOVA y ANCOVA } \\
\text { Mejora significativa para el grupo inter vención en el } \\
\text { número de estadios completados en el test de } 20 \text { metros } \\
(p<.001) \text { y mejoras significativas en la estimación del } \\
\text { VO2 máx }(p<.001)\end{array}$ \\
\hline $\begin{array}{l}\text { Valero-Valenzuela, } \\
\text { García, Camerino \& } \\
\text { Manzano (2020) }\end{array}$ & $\begin{array}{l}\text { Secundaria } \\
\text { Murcia } \\
\text { (Murcia) }\end{array}$ & $\begin{array}{l}2^{\circ} \text { y } 3^{\circ} \text { ESO } \\
\text { Educación Física } \\
10 \text { sesiones } \\
n=55\end{array}$ & $\begin{array}{l}\text { Autonomía, } \\
\text { responsabilidad y } \\
\text { motivación }\end{array}$ & $\begin{array}{c}\text { Sistema de observación personal } \\
\text { y responsabilidad social (SORPS) } \\
+ \\
\text { Escala de motivación en } \\
\text { educación secundaria (EME-S) }\end{array}$ & $\begin{array}{l}\text { Técnica detección patrones temporales y análisis } \\
\text { descriptivo e inferencial } \\
\text { Disminución significativa de la amotivación } \\
\qquad(p<.1) \\
\text { Aumento de } 1.12 \text { puntos en la autodeterminación }\end{array}$ \\
\hline
\end{tabular}


ma habitual en las aulas de infantil y también en primaria, aprovechando la curiosidad y creatividad que presenta el alumnado en estas etapas (Cañal de León, GarcíaCarmona \& Cruz-Guzmán, 2016; Márquez \& Abundez, 2015; Zacatelco, Chávez \& González, 2016). Esto puede ser consecuencia de que para muchos docentes no universitarios la investigación es un saber teórico que se aleja de la realidad del aula, convirtiéndose en algo no prioritario para ellos (Perines \& Murillo, 2017). Sin embargo, sí lo es para los docentes universitarios, a lo que además habría que añadir los altos índices de desmotivación y pasividad que existen en dicha etapa (Mira, Parra-Meroño y Beltrán, 2017; Nolasco \& Moradelli, 2009). Por tanto, parece lógico que en la docencia universitaria se necesiten programas que apuesten por la motivación y el aprendizaje significativo que propician las experiencias de gamificación, en la línea de otras revisiones realizadas en el marco internacional como, por ejemplo, la de Borges, Durelli, Reis \& Isotani (2014).

La motivación, de hecho, es la principal variable a la que han atendido las diferentes propuestas de gamificación, sin diferencias entre las diversas etapas educativas, lo que denota los enormes problemas motivacionales que encontramos en la actualidad en el ámbito educativo (Bolívar, 2018; Torres, 2016; Zepeda et al., 2016). Junto a ella, la mejora de hábitos saludables representa la variable que más se atiende en los artículos que componen esta revisión sistemática. Esto es consecuencia, evidentemente, de los niveles tan alarmantes que hay de sedentarismo y obesidad en la sociedad actual, tanto en España con fuera de sus fronteras (Black et al., 2017; Langford et al., 2018). Sin embargo, sería muy recomendable que en dichas intervenciones se hubiera incluido un retest para comprobar el grado de asentamiento de los hábitos alcanzados tras la finalización del programa.

A pesar de que desde el año 2011 se encuentran referencias internacionales de propuestas gamificadas (Borges et al., 2014; Hamari et al., 2014), en nuestro país no se empiezan a llevar a cabo hasta el 2014. De hecho, en los dos últimos años, han tenido lugar el 40\% de ellas. Por tanto, la tendencia sugiere que se trata de una estrategia metodológica cada vez con más protagonismo en las aulas españolas.

Otro aspecto a destacar tras el análisis realizado es la confirmación de la gran confusión terminológica y conceptual existente. Son numerosos los trabajos en los que se considera de forma errónea a los juegos educativos y al aprendizaje basado en juegos como manifesta- ciones de la gamificación, circunstancia que ya señalaba Majuri et al., (2018), y que ha dado lugar a excluir en torno a un tercio de los artículos una vez leídos en su totalidad.

Por otro lado, el 14\% que pasaba el primer filtro se acabó descartando por ser PBL. Se han excluido estas propuestas dado que, como sostienen Fischer, Heinz, Schlenker \& Follert (2016), la gamificación es un proceso más abstracto, complejo y estratégico que pretende ir más allá del uso de puntos, insignias y clasificaciones. Y más aún si la finalidad es generar hábitos perdurables en el tiempo o favorecer la motivación intrínseca hacia el aprendizaje. Es más, en una experiencia gamificada la narrativa es fundamental, ya que favorece la sensación de inmersión en la actividad (Hamari, Shernoff, Rowe, Coller, Asbell-Clarke \& Edwards, 2016). De hecho, gracias a ella se podrá incrementar la motivación de los estudiantes y conectar los contenidos entre sí, dando sentido a lo que se está trabajando en cada momento y ofreciendo una sensación de continuidad e inmersión (Cornellà \& Estebanell, 2018; Ortiz-Colón et al., 2018; Perrotta, Featherstone, Aston \& Houghton, 2013). De este modo se logrará una experiencia más significativa que potenciará que el alumnado quiera profundizar en ella y mejorar su aprendizaje (Quintero-González, et al., 2018).

La evaluación que se ha llevado a cabo en las distintas propuestas se caracteriza por ser genérica y poco rigurosa, utilizando en la mayoría de casos herramientas que no están validadas. Esta circunstancia puede estar debida a las dificultades que señalan diversos autores para evaluar las propuestas gamificadas como consecuencia de la falta de frameworks que faciliten un diseño más claro y formal (Díez, Bañeres \& Serra, 2017). La variable motivación es un ejemplo claro de este problema. En lugar de utilizar instrumentos con la suficiente fiabilidad y validez como para determinar la incidencia que la propuesta gamificada tiene sobre ella, se suele establecer una correspondencia poco rigurosa que relaciona una mejora del rendimiento académico con la motivación, sin tener en cuenta el resto de variables que puedan incidir en ello. De igual modo, tampoco suele complementarse dicho análisis con una evaluación cualitativa que permitiera valorar con una mayor profundidad la incidencia real de la intervención.

A todo ello hay que sumarle que en muchos de los trabajos no se especificaba con detalle el tipo de diseño que se implementó, ni se realizaba una evaluación inicial con la que poder cotejar los resultados obtenidos al término de la experiencia, ni se contaba con un grupo 
control donde se desarrollara un planteamiento tradicional de enseñanza que permitiera valorar la repercusión real de la propuesta gamificada. De igual modo, salvo en determinados casos, no se especifica ni el número de sesiones con las que contaba el programa, ni el enfoque concreto de las mismas. Circunstancias todas estas que no solo dificultan una adecuada interpretación de los resultados obtenidos, sino que, además, reducen significativamente la posibilidad de replicar dichas intervenciones.

\section{Conclusión}

Tras todo lo analizado resulta evidente la necesidad de seguir investigando en esta línea, ya que está teniendo un gran auge a nivel internacional y, sin embargo, se encuentran pocos trabajos empíricos en nuestro país. Además, es preciso mejorar en ellos tanto los planteamientos metodológicos como una descripción más exhaustiva de las propuestas en cuestión. Del mismo modo, resultará crucial una mayor rigurosidad en los procesos de revisión de los artículos que acaban publicándose en revistas científicas para, de este modo, evitar la confusión terminológica que existe al respecto.

Al encontrar una gran disparidad en el tamaño de la muestra, intervalo de aplicación, la metodología desarrollada, las variables sobre las que se incide y los instrumentos de medida, es complicado realizar un análisis lo suficientemente clarificador de la situación o estado de la gamificación en España. Por tanto, no se pueden extraer conclusiones concluyentes y extrapolables. Sin embargo, sí hay indicios que muestran el potencial de la gamificación en el ámbito educativo español. En todos los trabajos que se incluyen en esta revisión sistemática se obtuvieron mejoras en alguna de las variables evaluadas, destacando en especial su incidencia sobre la motivación del alumnado y el desarrollo de parámetros relacionados con su salud.

Como consecuencia del reducido número de artículos existentes que cumplían con los criterios establecidos, en futuras revisiones sería interesante completar la búsqueda ampliando a un mayor número de bases de datos que abarquen revistas de índole más divulgativo. De este modo, se podrá tener una idea más exacta del trabajo realizado en la actualidad y, en especial, en las aulas de infantil y primaria. También sería de gran valor analizar la repercusión sobre las variables motivacionales y de rendimiento académico de un sistema PBL en comparación con una experiencia de gamificación.

\section{Referencias}

Arnold, B.J. (2014). Gamification in education. Proceedings of the American Society of Business and Behavioral Sciences, 21(1), 32-39.

Black, M.M., Walker, S.P., Fernald, L.C.H., Andersen, C. T., DiGirolamo, A M., Lu, C., McCoy, D.C., Fink, G., Shawar, Y.R., Shiffman, P.J., Devercelli, A.E., Wodon, Q.T., Vargas-Bar., \& GranthamMcGregor, S. (2016). Early childhood development coming of age: science through the life course. Lancet, 6736, 1-14.

Bolívar, A. (2018). Nueva gobernación en educación y dinámicas para la mejoría: presión versus compromiso. En M.L. Pinto de Almeida, M. Pasqual y J.Moreles (coord.), Estado, Políticas Públicas y Gobernación (pp. 23-57). CLACSO: Mercado de Letras.

Bonilla, A. (2015). Diseño de juegos y creatividad: un estudio en el aula universitaria. Opción: Revista de Ciencias Humanas y Sociales, 31(4), 106-126.

Borges, S.S., Durelli, V.H.S, Reis, H.M., \& Isotani, S. (2014, marzo). A systematic mapping on gamification applied to education. En Proceedings of the 29th annual ACM symposium on applied computing. (pp. 216-222). Association for Computer Machinery, Gyenongju, Korea.

Buckley, P., Doyle, E., \& Doyle, S. (2017). Game On! Students' Perceptions of Gamified Learning. Journal of Educational Technology y Society, 20(3), 1-10.

Cañal de León, P., García-Carmona,A., \& Cruz-Guzmán, M. (2016). Didáctica de las Ciencias Experimentales en Educación Primaria. Madrid, España: Ediciones Paraninfo S.A.

Cornellà, P., \& Estebanell, M. (2018). GaMoodlification: Moodle al servicio de la gamificación del aprendizaje. En C.S. González (coord.), Actas del Congreso Internacional de Videojuegos y Educación (CIVE'17). Universidad de La Laguna, San Cristóbal de La Laguna, Tenerife, España.

Chang, T.P., Raymond, T., Dewan, M., Whitfill, T., Harwayne-Gidansky, I., Doughty, C. B., Frisell, K., Kessler, D.O., Wolfe, H., Auerbach, M.A., Rutledge, C., MacKinnon, R., Mitchell, D., Jani, P., \& Walsh, C.M. (2018). The Effect of Competitive Gamification on Self-Directed CPR Training Among Healthcare Professionals: A Multi-National Randomized-Control Study. Circulation, 138(2), 273-281.

Chrobak, R. (2017). El aprendizaje significativo para fomentar el pensamiento crítico. Archivos de Ciencias de la Educación, 11(12). 
De Soto-García, I.S. (2018). Herramientas de gamificación para el aprendizaje de ciencias de la tierra. Revista Electrónica de Tecnología Educativa, 65, 29-39.

Del Moral, M., Guzmán-Duque, A., \& FernándezGarcía, L. (2018). Game-based learning: Increasing the logical-mathematical, naturalistic, and linguistic learning levels of primary school students. Journal of New Approaches in Educational Research (NAER Journal), 7(1), 31-39.

Deterding, S. (2011). Situated motivational affordances of game elements: a conceptual model. En Gamification: Using Game Design Elements in NonGamingContexts, a Workshop at CHI (pp. 1-4). ACM, Vancouver, Canadá.

Dichev, C., Dicheva, D., Angelova, G., \& Agre, G. (2014). From Gamification to Gameful Design and Gameful Experience in Learning. Cybernetics and Information Technologies, 14(4), 80-100.

Dicheva, D., Dichev, C., Agre, G., \& Angelova, G. (2015). Gamification in education: A systematic mapping study. Journal of Educational Technology \& Society, 18(3), 75-88.

Díez, J.C., Bañeres, D., \& Serra, M. (2017). Experiencia de Gamificacioin en Secundaria en el Aprendizaje de Sistemas Digitales. Education in the Knowledge Society, 18(2), 85-105.

Faiella, F., \& Ricciardi, M. (2015). Gamification and learning: a review of issues and research. Journal of e-Learning and Knowledge Society, 11(3), 13-21.

Fernandez-Río, J., De las Heras, E., González, T., Trillo, V., \& Palomares, J. (2020). Gamification and physical education. Viability and preliminary views from students and teachers. Physical Education and Sport Pedagogy, 25(5), 509-524.

Fischer, H., Heinz, M., Schlenker, L., \& Follert, F. (2016, junio). Gamifying higher education. Beyond badges, points and Leaderboards. En Knowledge Communities in Online Education and (Visual) Knowledge Management. (pp. 93-105). GeNeMe, Dresden, Alemania.

Garrote, D., Garrote, C., \& Jiménez-Fernández, S. (2016). Factores influyentes en motivación y estrategias de aprendizaje en los alumnos de grado. Revista Iberoamericana sobre Calidad, Eficacia y Cambio en Educación, 14(2), 31-44.

Giménez-Giubbani, A. (2016). El papel de la gestión de centros educativos en un modelo de aprendizaje basado en competencias. Páginas de Educación, 9(1), 515.

Gómez-Gonzalvo, F., Molina-Alventosa, P., \& Devis, J.
(2018). Los videojuegos como materiales curriculares: una aproximación a su uso en Educación Física. Retos, 34, 305-310.

Hamari, J. (2013). Transforming homo economicus into homo ludens: A field experiment on gamification in a utilitarian peer-to-peer trading service. Electronic commerce research and applications, 12(4), 236-245.

Hamari, J., Shernoff, D. J., Rowe, E., Coller, B., AsbellClarke, J., \& Edwards, T. (2016). Challenging games help students learn: An empirical study on engagement, flow and immersion in game-based learning. Computers in human behavior, 54, 170-179.

Hamari,J., Koivisto,J., \& Sarsa, H. (2014, enero) Does Gamification Work? A Literature Review of Empirical Studies on Gamification. En Hawaii International Conference on System Sciences (pp. 30253034). IEEE, Waikoloa, Hawai.

Hanus, M.D., \& Fox, J. (2015). Assessing the effects of gamification in the classroom: A longitudinal study on intrinsic motivation, social comparison, satisfaction, effort, and academic performance. Computers and Education, 80, 152-161.

Jiménez-Serrano, Ó. (2002). Methodological and professional challenges posed by new technologies in the teaching of technical translation. En Simposi sobre l'Ensenyament a distància i semipresencial de la Tradumàtica, (pp. 1-6). Universidad Autónoma de Barcelona.

Kapp, K.M. (2012). The Gamification of Learning and Instruction:Game-based Methods and Strategies for Training and Education. San Francisco, California: John Wiley $\&$ Sons.

Langford, R., Bonell, C., Jones, H., Pouliou, T., Murphy, S., Waters, E.,... \& Campbell, R. (2015). The World Health Organization's Health Promoting Schools framework: a Cochrane systematic review and meta-analysis. BMC public health, 15(1), 130.

Lee, J.J., \& Hammer, J. (2011). Gamification in education: What, how, why bother? Academic exchange quarterly, 15(2), 146.

León-Díaz, Ó., Martínez-Muñoz, L.F., \& Santos-Pastor, M.L. (2019). Gamificación en Educación Física: un análisis sistemático de fuentes documentales. Revista Iberoamericana de Ciencias de la Actividad Física y el Deporte, 8(1), 110-124.

Llopis, M.A. \& Balaguer, P. (2016). El uso del juego en educación. Gamificación. En Chiva, O. y Martí, M. (coords), Métodos pedagógicos activos y globalizadores. Conceptualización y propuestas de aplicación, (pp. 85-102). Barcelona, España: Graó. 
López-López, M.M. (2019). La pedagogía crítica como propuesta innovadora para el aprendizaje significativo en la educación básica. ReHuSo: Revista de Ciencias Humanísticas y Sociales, 4(1), 87-98.

Lumsden, J., Edwards, E.A., Lawrence, N.S., Coyle, D. , \& Munafò, M.R. (2016). Gamification of cognitive assessment and cognitive training: a systematic review of applications and efficacy. JMIR serious games, 4(2).

Majuri, J., Koivisto, J., \& Hamari, J. (2018, mayo). Gamification of education and learning: A review of empirical literature. En Proceedings of the 2nd International GamiFIN Conference (pp 11-19). CEUR, Pori, Finlandia.

Marczewski, A. (2018). Gamification. Even Ninja Monkeys Like to Play (Unicorn Edition). Gamified UK.

Marín, I. (2018). jugamos? Madrid, España: Ediciones Paidós.

Márquez, M.L., \& Abundez, M.A. (2015). La motivación en el aula: estrategia esencial para mejorar el aprendizaje en la escuela primaria. Cuadernos de Educación y Desarrollo, 55, 1-10.

Martín-Moya, R., Ruiz-Montero, P.J., Chiva-Bartoll, Ò., \& Capella-Peris, C. (2018). Motivación de logro para aprender en estudiantes de educación física: Diverhealth. Revista Interamericana de Psicología, 52(2), 270-280

Menchaca, S., Moya, A., \& Bastida, L. (2019, octubre). A gamified Experience for Motivating Students to Learn Literature. En 3rd International Symposium on Gamification and Games for Learning (GamiLearn'19). CEUR Workshop Proceeding, Barcelona, España.

Mira, J.G., Parra-Meroño, M.C., \& Beltrán, M.A. (2017). Emotional education in the University: proposed activities for development of the social and personal abilities. Vivat Academia. Revista de Comunicación, 139, 1-17.

Monguillot, M., González-Arévalo, C., Zurita, C., Almirall, L., \& Guitert, M. (2015). Play the Game: gamification and healthy habits in physical education. Apunts. Education Física and sports, 119, 71 79.

Mora, A., Riera, D., González, C., \& Arnedo-Moreno, J. (2017). Gamification: a systematic review of design frameworks. Journal of Computing in Higher Education, 29(3), 516-548.

Mora-González, J., Pérez-López, I.J., \& DelgadoFernández, M. (2020). The «\$inTIME» Gamification Project: Using a Mobile App to Improve Cardiorespiratory Fitness Levels of College
Students. Games for health journal, 9(1) 37-44.

Mora-González, J., Pérez-López, I.J., Esteban-Cornejo, I., \& Delgado-Fernández, M. (2020). A Gamification-Based Intervention Program that Encourages Physical Activity Improves Cardiorespiratory Fitness of College Students: 'The Matrix rEFvolution Program'. International Journal of Environmental Research and Public Health, 17(3), 877.

Nah, F.F.H., Zeng, Q., Telaprolu, V.R., Ayyappa, A.P., \& Eschenbrenner, B. (2014, junio). Gamification of education: a review of literature. In International conference on hci in business (pp. 401-409). Suiza: Springer.

Nolasco, M.R., \& Modarelli, M.C. (2009). Metodología didáctica innovadora: una experiencia en el aula universitaria. Revista iberoamericana de educación, 48(2), 1-8.

Ortega-Navas, M.C. (2016). La formación del carácter en los futuros maestros como proceso educativo orientado hacia la democratización educativa. En I. Carrillo (coord.), Democracia y Educación en la formación docente, (pp. 227-231). Barcelona, España: Universidad Centra del Cataluña.

Ortiz-Colón, A.M., Jordán, J., \& Agredal, M. (2018). Gamification in education: an overview on the state of the art. Educação e Pesquisa, 44, 2-17.

Parra-González, M.E., \& Segura-Robles, A. (2019). Análisis de las experiencias gamificadas de docentes y alumnos de Educación Secundaria. Revista Espacios, 40(23), 15-25.

Peirez-Loìpez, I.J., Rivera-Garciia, E., \& Trigueros, C. (2019). 12+ 1. Sentimiento del alumnado universitario de educación física frente a una propuesta de gamificacioin:"Game of thrones: la ira de los dragones”. Movimento, 25, 1-15.

Pérez-López, I.J., Rivera, E., \& Delgado-Fernández, M. (2017). Mejora de hábitos de vida saludables en alumnos universitarios mediante una propuesta de gamificación. Nutrición hospitalaria, 34(4), 942-951.

Pérez-López, I.J., Rivera, E., \& Trigueros, C. (2017). La profecía de los elegidos: un ejemplo de gamificación aplicado a la docencia universitaria. Revista Internacional de Medicina y Ciencias de la Actividad Física y del Deporte, 17(66), 243260.

Perines, H., \& Murillo, F.J. (2017). ¿Cómo mejorar la investigación educativa? Sugerencias de los docentes. Revista de la educación superior, 46(181), 89104.

Perrenoud, P. (2012). Cuando la escuela pretende preparar 
para la vida. Barcelona, España: Graó.

Perrotta, C., Featherstone, G., Aston, H., \& Houghton, E. (2013). Game-based learning: Latest evidence and future directions. Slough, Inglaterra: National Foundation for Educational Research.

Quintero-González, L., Jiménez-Jiménez, F., \& AreaMoreira, M. (2018). Más allá del libro de texto. La gamificación mediada con TIC como alternativa de innovación en Educación Física. Retos, 34, 343-348.

Sánchez-Martín, J., Cañada-Cañada, F., \& Dávila-Acedo, M.A. (2017). Just a game? Gamifying a general science class at university: Collaborative and competitive work implications. Thinking Skills and Creativity, 26, 51-59.

Severín, E. (2017). Un nuevo paradigma educativo. Educación y ciudad, 32, 75-82.

Sousa-Vieira, M.E., López-Ardao, J.C., FernándezVeiga, M., Rodríguez-Pérez, M., \& López-García, C. (2015). Using social learning methodologies in higher education. International Journal of Engineering Pedagogy, 5(2), 64-72.

Tobón, S., Guzmán, C.E., Silvano-Hernández, J., \& Cardona, S. (2015). Sociedad del conocimiento: Estudio documental desde una perspectiva humanista y compleja. Paradigma, 36(2), 7-36.

Toledo, P., Toda, A.M., Oliveira, W., Cristea, A. I., \& Isotani, S. (2019, julio). Narrative for gamification in education: why should you care?. En 19th International Conference on Advanced Learning Technologies (ICALT) (pp. 97-99). IEEE, Maceió, Brazil.

Torres, M. (2016). Factores de desmotivación más sobresalientes en el aprendizaje: la afectividad requisito ineludible. En $5^{\circ}$ Congresso Iberoamericano em Investigação Qualitativa em Educação, (pp. 732-735). Universidade Lusófona do Porto, Oporto, Portugal. Torres, A., Romero-Rodríguez, L., Pérez-Rodríguez, M.A. \& Björk, S. (2018). Modelo Teórico Integrado de Gamificación en Ambientes E-Learning (EMIGA). Revista Complutense de Educación, 29(1), 129. 145

Valero-Valenzuela, A., García, D.G., Camerino, O., \& Manzano, D. (2020). Hibridación del modelo pedagógico de responsabilidad personal y social y la gamificación en educación física. Apunts. Educación física y deportes, 3(141), 63-74.

Vicent, L., Villagrasa, S., Fonseca, D., \& Redondo, E. (2015). Virtual learning scenarios for qualitative assessment in higher education 3D arts. Journal of universal computer science, 21(8), 1086-1105.

Villagrasa, S., Fonseca, D., Redondo, E., \& Durán, J. (2014, octubre). Teaching case: applying gamification techniques and virtual reality for learning building engineering 3D arts. En Proceedings of the second international conference on technological ecosystems for enhancing multiculturality (pp. 171-177). Association for Computing Machinery, Salamanca, España.

Zacatelco, F., Chávez, B., \& González, A. (2016). Factores que influyen en los juicios del profesor, para nominar estudiantes en educación básica. En J.L. Castejón (coord.), Psicología y educación: Presente y futuro (pp. 1367-1374). Asociación Científica de Psicología y Educación.

Zainuddin, Z., Chu, S.K.W., Shujahat, M., \& Perera, C.J. (2020). The impact of gamification on learning and instruction: A systematic review of empirical evidence. Educational Research Review, 30.

Zepeda-Hernández, S., Abascal-Mena, R., \& LópezOrnelas, E. (2016). Análisis cualitativo de experiencias y emociones de los alumnos en el aula. $R a$ Ximhai, 12(6), 347-358.

Zichermann, G., \& Cunningham, C. (2011). Gamification by design: Implementing game mechanics in web and mobile apps. Sebastopol, California: O’Reilly Media, Inc.

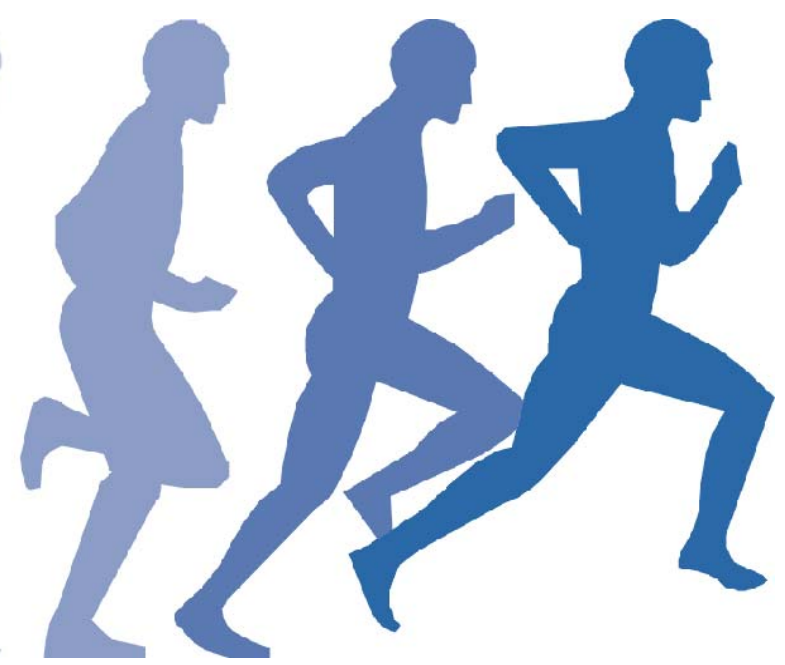

